\title{
WARPED GALAXIES FROM MISALIGNED ANGULAR MOMENTA
}

\author{
Victor P. Debattista ${ }^{1}$ and J. A. Sellwood \\ Department of Physics and Astronomy, Rutgers, The State University of New Jersey, 136 Frelinghuysen Road, \\ Piscataway, NJ 08854-8019; debattis@ astro.unibas.ch, sellwood@physics.rutgers.edu \\ Received 1998 August 17; accepted 1999 January 12; published 1999 February 11
}

\begin{abstract}
A galaxy disk embedded in a rotating halo experiences a dynamical friction force which causes it to warp when the angular momentum axes of the disk and halo are misaligned. Our fully self-consistent simulations of this process induce long-lived warps in the disk that mimic Briggs's rules of warp behavior. They also demonstrate that random motion within the disk adds significantly to its stiffness. Moreover, warps generated in this way have no winding problem and are more pronounced in the extended $\mathrm{H}$ I disk. As emphasized by Binney and his coworkers, angular momentum misalignments, which are expected in hierarchical models of galaxy formation, can account for the high fraction of warped galaxies. Our simulations exemplify the role of misaligned spins in warp formation even when the halo density is not significantly flattened.

Subject headings: galaxies: evolution — galaxies: halos — galaxies: kinematics and dynamics galaxies: structure — radio lines: galaxies
\end{abstract}

\section{INTRODUCTION}

An "integral sign" twist has been observed in the extended $\mathrm{H}$ I disk of many galaxies; in some cases, it can also be seen in the star light. Briggs (1990) characterized the behavior of a sample of 12 warped galaxies as (1) coplanar inside $R_{25}$, and warped beyond, with a straight line of nodes (LON) inside the Holmberg radius $\left(R_{\mathrm{Ho}}\right)$, (2) changing near $R_{\mathrm{Ho}}$, (3) into a LON on a leading spiral (as seen from the inner disk) outside $R_{\mathrm{Ho}}$. Bosma (1991) found 12 clearly warped disks in a sample of 20 edge-on systems; taking into account random warp orientation, the true fraction of warped disks must be larger. This high fraction of warped galaxies implies either that warps are long-lived features or that they are repeatedly regenerated.

If a twisted disk were modeled as a set of uncoupled tilted rings in a flattened potential, their changing precession rates with radius would lead to a winding problem, similar to that for spirals (e.g., Binney \& Tremaine 1987). If warps are longlived, therefore, some means to overcome differential precession is required (see reviews by Toomre 1983 and Binney 1992).

Most recent ideas for warp formation rely in some way on the influence of the halo. Toomre (1983) and Dekel \& Shlosman (1983) suggested that a flattened halo misaligned with the disk can give rise to a warp, and Toomre (1983), Sparke \& Casertano (1988), and Kuijken (1991) found realistic warp modes inside rigid halos of this form. However, angular momentum conservation requires there to be a back-reaction on the halo (Toomre 1983; Binney 1992); Dubinski \& Kuijken (1995) and Nelson $\&$ Tremaine (1995) showed that a mobile halo should cause a warped disk to flatten quickly (but see also Binney, Jiang, \& Dutta 1998).

Since a warp represents a misalignment of the disk's inner and outer angular momenta, Ostriker \& Binney (1989) proposed a qualitative model in which the warp is generated by the slewing of the galactic potential through accretion of material with misaligned spin. The accretion of satellites by larger galaxies, such as in the Milky Way, provides direct evidence of late-infalling material with misaligned angular momentum.

${ }^{1}$ Current address: Astronomisches Institut, Universität Basel, Venusstrasse 7, CH-4102 Binningen, Switzerland.
Such misalignments are expected to be generic in hierarchical models of galaxy formation (Quinn \& Binney 1992) in which the spin axis of late-arriving material, both clumpy and diffuse, is poorly correlated with that of the material that collapsed earlier. Jiang \& Binney (1999) calculate a concrete example of a warp formed through the addition of a misaligned torus of matter.

If the accreted material is flattened due to its intrinsic spin, a density misalignment is present immediately and exerts a torque to twist the disk, as in Jiang \& Binney's model. But dissipationless halo material may not be strongly flattened despite streaming about a misaligned axis. Nevertheless, such material will exert a torque on the disk through dynamical friction, causing the disk's angular momentum vector to tip toward alignment with that of the halo, as we show in this Letter.

Rotation in the inner halo will cause the disk to tilt differentially because the inner disk experiences a stronger dynamical friction torque (since the densities of both disk and halo are highest) and because timescales are shortest in the center. As the inner disk begins to tip, the usual gravitational and pressure stresses in a twisted disk become important. Our fully selfconsistent $N$-body simulations show that this idea is actually quite promising and leads to warps that are relatively longlived and of the observed form.

Dynamical friction arises through an aspherical density response in the halo that lags "downstream" from the disk. In the long run, however, the density distribution in the halo must become symmetric about the disk plane, even while it continues to rotate about a misaligned axis. Simple time-reversibility arguments dictate that a steady system cannot support net torques (see the "anti-spiral" theorem: Lynden-Bell \& Ostriker 1967; Kalnajs 1971). We therefore find that the dynamical friction torque on the disk does not persist indefinitely. We do not regard this as a serious objection to our model, since late-infalling material must constantly revise the net angular momentum of the halo.

\section{NUMERICAL METHOD}

Since mild force anisotropies in many grid-based $N$-body methods can cause an isolated disk to settle to a preferred plane 


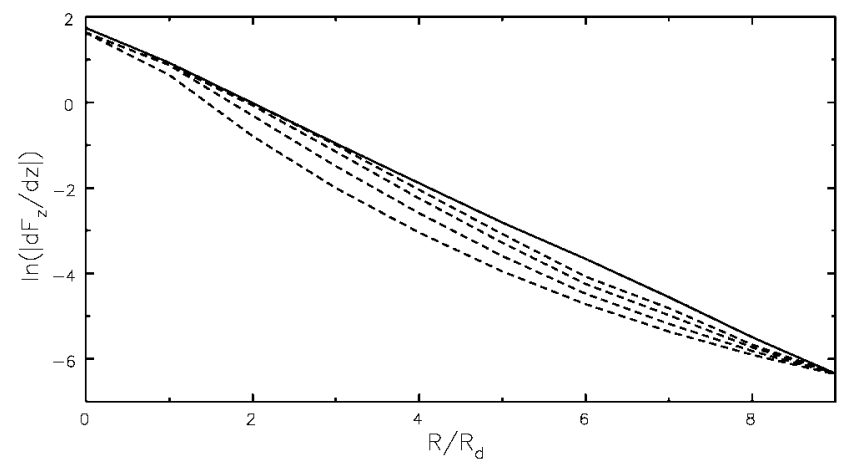

FIG. 1.-Midplane force gradient for the disk described in $\S 4$. The solid line is the exact gradient, and the dashed lines show values obtained with $l_{\max }=10,20,35$, and 50 in order of decreasing error.

(e.g., May \& James 1984), we adopt a code with no preferred plane. An expansion of the potential in spherical harmonics has been widely used for $N$-body simulations both with a grid (van Albada 1982) and without (Villumsen 1982; White 1983; McGlynn 1984). Here we adopt an intermediate approach: we tabulate coefficients of a spherical harmonic expansion of the density distribution on a radial grid and interpolate for the gravitational forces between the values on these shells. The radial grid smooths the gravitational field, thereby avoiding the problem of "shell crossings." Since there is no gridding in the angular directions, we retain the full angular resolution up to the adopted $l_{\max }$ - the maximum order of the spherical harmonic expansion.

While avoiding a preferred plane, this method is not well suited to representation of disks. The vertical restoring force to the disk midplane converges slowly with increasing $l_{\max }$, as shown in Figure 1. Most of our simulations included terms to $l_{\max }=10$ only; tests with higher $l_{\max }$ (and fewer particles) suggest that these models overestimate the magnitude and duration of the warp in the massive part of the disk, although milder and shorter-lived warps still develop. Moreover, the warp in the test-particle layer beyond the edge of the massive disk is unaffected by force resolution.

\section{INITIAL MODEL}

While we have found that a massive halo is able to produce spectacular warping, we here prefer more realistic minimal halo models (see Debattista \& Sellwood 1998). Our galaxy model has three massive components: an exponential disk of lengthscale $R_{d}$ truncated at $8 R_{d}$, a polytropic halo, and a central softened point mass. The disk:halo:central mass ratio is 1:9:0.2, chosen to give a roughly flat rotation curve out to $\sim 15 R_{d}$. We also include a disk of test particles, extending well outside the massive disk, that is intended to mimic the neutral hydrogen layer of a galaxy.

The initial massive disk had velocity dispersions set by adopting Toomre $Q=1.5$ and a thickness of $0.1 R_{d}$, which were both independent of radius. The massless particles started with exactly circular orbits in the disk midplane. The central mass is a single particle with a core radius of $0.3 R_{d}$.

The initial distribution of halo particles was generated by the method first used by Raha et al. (1991), which gives an exact equilibrium isotropic halo in the potential of the disk and central mass. The halo extends to a radius $r_{\text {trunc }}=30 R_{d}$. Because the disk has mass, the halo is not precisely spherical; its

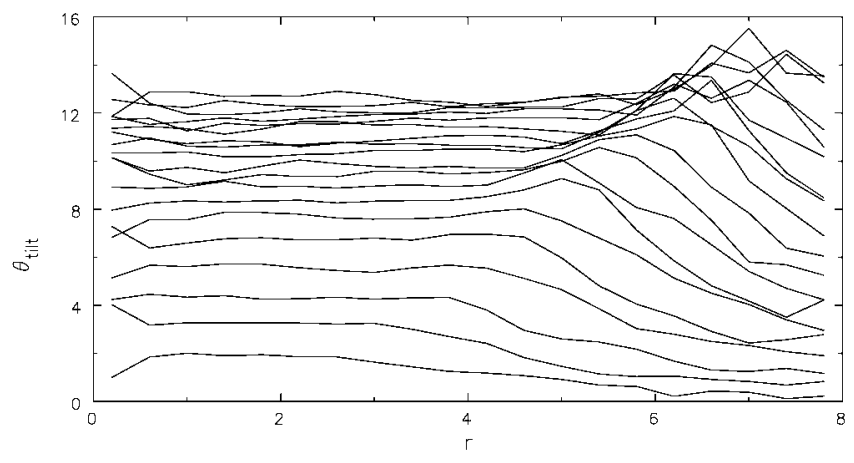

FIG. 2.-Disk tilt angle (in degrees) as a function of radius at intervals of 50 time units ( $t=50$ to $t=950$ from bottom up).

initial axis ratio varies from closely spherical at $r \geq 4 R_{d}$ to $c / a \simeq 0.7$ near $r=1.5 R_{d}$. The initial halo angular momentum was created by selectively reversing halo particle velocities about a chosen axis, which for run 1 is tipped away from the disk spin (z-)axis by $45^{\circ}$ in the $x$-direction. We chose a value of the dimensionless spin parameter $\lambda \equiv(L / G)(|E|$ $\left(M^{5}\right)^{1 / 2} \simeq 0.07$ for our halo models; here $L, E$, and $M$ are respectively the total angular momentum, energy, and mass of the halo. A value of 0.07 is typical in hierarchical clustering models (Barnes \& Efstathiou 1987; Steinmetz \& Bartelmann 1995).

We work in units where $G=M\left(=M_{\text {disk }}+M_{\text {halo }}\right)=R_{d}=$ 1 ; the unit of time is therefore $\left(R_{d}^{3} / G M\right)^{1 / 2}$. A rotation period in the disk plane at $R=1$ is 32 . We set $l_{\max }=10$ and used a radial grid with 200 shells and a time step of 0.05 . The disk and halo components are represented by a total of $10^{6}$ equal mass particles. Our simulations conserve energy to better than $0.04 \%$.

\section{RESULTS}

\subsection{Warp in the Massive Disk}

The outer disk lags as the inner part of the disk in run 1 begins to tilt, causing a warp to develop almost at once. Figure 2 shows that the approximately rigid tilt of the inner disk increases rapidly at first and then more slowly, while the radius at which the warp starts also moves outward over time. The disk in run 1 at $t=400$ is shown in Figure 3.

Figure 4 shows the warp of the massive disk in the form of a Tip-LON diagram (Briggs 1990) at equally spaced times. Each point represents the direction of the normal to the best-

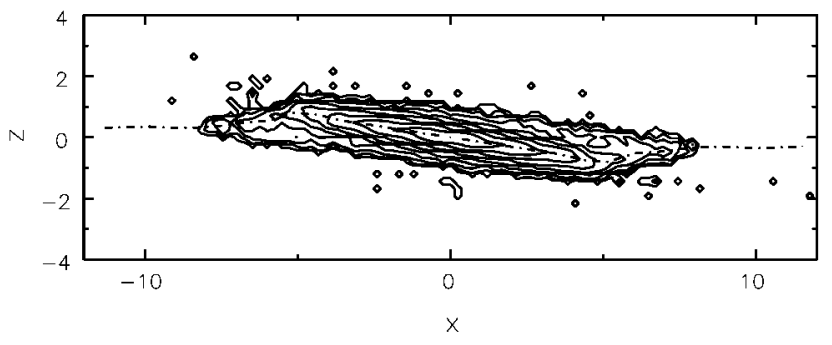

FIG. 3.-Contours of the disk density in run 1 at $t=400$ projected onto the $(x, z)$-plane. The dot-dashed line indicates the cross section of the layer of massless particles. By this time, the inner disk has tilted $\sim 10^{\circ}$ away from its original plane, which was horizontal. 


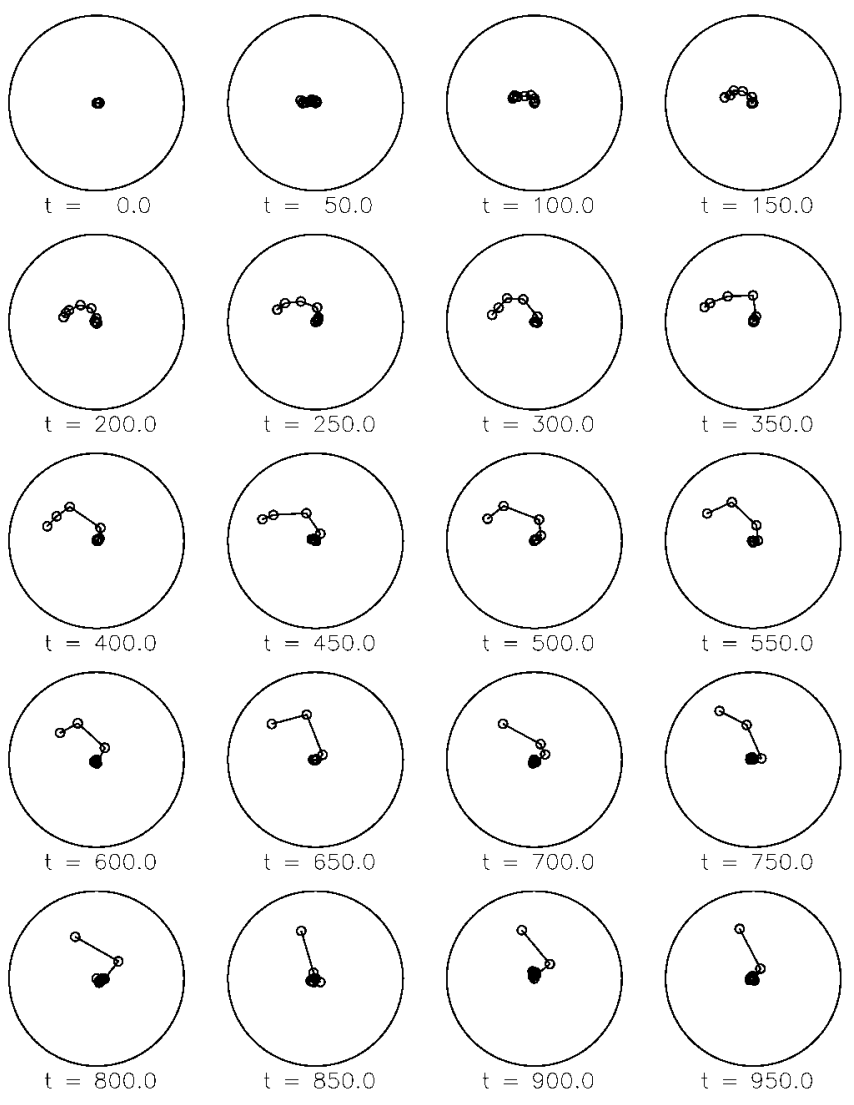

FIG. 4.-Polar angle plots showing the shape of the warped disk. The points indicate the angles $(\theta, \phi)$ of the symmetry axis of an annular piece of the disk (width $0.8 R_{d}$ ) relative to the inner disk axis at the given time. The radial coordinate is the polar angle $\theta$, and the azimuthal coordinate shows the azimuth $\phi$ of the LON of each annulus. The boundary circle is at $\theta=10^{\circ}$. Disk rotation is counterclockwise.

fit plane of an annular piece of the disk, with the center of the disk defining the origin. The normal to the inner disk tilts initially in the $(x, z)$-plane, while the outer disk is left behind, thereby shifting outer-disk points along the $\phi=180^{\circ}$ direction (e.g., $t=100$ ); the almost flat inner part of the disk gives rise to the concentration of points in the center. The warp reaches a maximum angle of $\sim 7^{\circ}$ at $t \simeq 350$, and it takes roughly 700 time units $\left(\sim 20\right.$ disk rotations at $\left.R=R_{d}\right)$ for most of the disk to settle back to a common plane.

The leading spiral, reminiscent of Briggs' (1990) third rule, develops through clockwise differential precession in the outer parts. Precession is a consequence of gravitational coupling between the inner and outer disk (Hunter \& Toomre 1969). The extremely slow precession of the outermost edge of the disk indicates that it is subject to a very mild torque, arising almost exclusively from the distant, tipped inner disk. The outer disk would precess more rapidly if the halo density distribution were flattened.

\subsection{Secular Evolution}

Several changes occur as the model evolves: the dynamical friction force driving the warp decays over time, as expected from $\S 1$, causing the inner disk to tilt more slowly (Fig. 2). The radius at which the warp starts moves outward, and the amplitude of the warp (the difference in tilt angle between the inner and outer disk) also decreases. The massive disk is almost

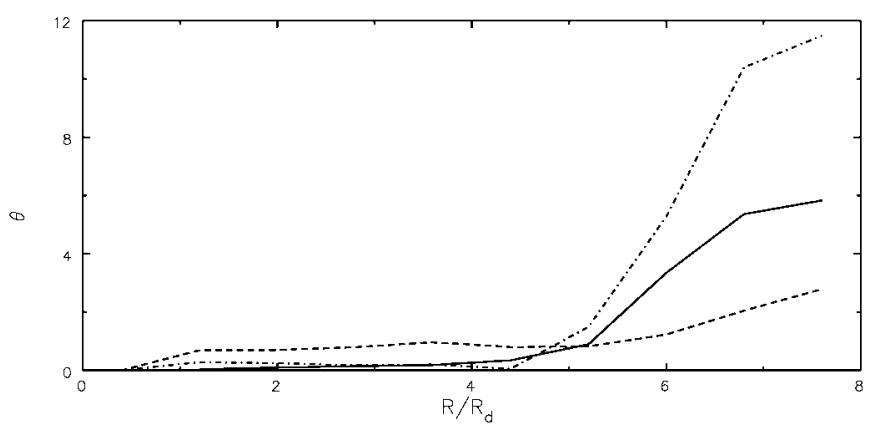

FIG. 5.-Warp angle as a function of radius for three different models. Solid line: our standard (run 1); dashed line: hotter disk (run 2); dot-dashed line: greater misalignment (run 3). All warp angles are at $t=450$.

coplanar again by time 1000 (Fig. 4). Spiral arms and a weak bar also drive up the velocity dispersion of the particles to $Q \simeq 2.5$.

The inner disk tilts remarkably rigidly, indicating strong cohesion which arises from two distinct mechanisms. Most studies have focused on gravitational forces, which Hunter \& Toomre (1969) found were inadequate to persuade the outer disk to precess along with the inner disk in a steady mode. However, the disk is also stiffened by the radial epicyclic excursions of the stars that communicate stresses across the disk.

Both self-gravity is strongest and random motion is greatest in the inner disk, where the coupling is evidently strong enough to preserve its flatness. The settling of the disk to ever larger radii should be describable in terms of the group velocity and/or damping of bending waves in a warm and finitely thick disk, but the absence of a dispersion relation valid in this regime precludes a comparison with theory. It is interesting that each annulus settles as its precession angle reaches $\sim 180^{\circ}$ (Fig. 4), thereby preventing excessive winding of the warp. The settling of each ring after half its precession period could be coincidental, but we have seen it in many models. One possible reason is that a warm disk cannot support bending waves with wavelength shorter than the average epicycle diameter; a prediction based on this idea is only roughly in accord with the radially dependent settling time, however. It should be noted that whether settling is described by group velocity, wave damping, or precession angles, it should be more rapid in a disk with stronger forces toward the midplane.

To demonstrate the importance of random motion, we ran a new simulation (run 2) identical to run 1 except with $Q=$ 4.0 initially in the disk. The warp was much reduced, as shown in Figure 5, even though the inner disk tilted by an angle comparable to that in run 1.

\subsection{Test-Particle Layer}

The sheet of test particles is intended to approximate a gaseous disk. Being massless, it does not induce a response from the halo, but is perturbed by forces from the tilted massive disk and its associated halo response. Within $8 R_{d}$, the test particles simply tilt or warp with the disk. Outside the massive disk, however, the disturbance forces from the halo and the massive disk drop off rapidly and the plane of this dynamically cool sheet hardly moves at large radii. It therefore appears warped relative to the plane of the tilted disk and remains so even by the end of the simulation, when the stellar disk has mostly settled. 


\subsection{Further Simulation}

The spin axis of the halo in run 1 was initially inclined at $45^{\circ}$ to that of the disk. In run 3 , we set this angle to $135^{\circ}$, thereby reversing the sign of $J_{z}$, the component of the halo's angular momentum along the $z$-axis. The larger misalignment angle causes the inner disk to tilt faster and further and gives rise to a larger warp, as shown in Figure 5. Dynamical friction also lasts longer. Other properties of the warp are similar to those in run 1 ; in particular, the warp begins at a similar radius at equal times.

\section{CONCLUSIONS}

Our simulations have confirmed that dynamical friction from a halo having angular momentum misaligned with that of the disk causes a transient warp. The warp has two properties commonly observed: the LON traces out a leading spiral relative to the inner disk and lasts longest in the $\mathrm{H}$ I layer.

By driving the warp, we side-step the most troublesome difficulties faced by other warp mechanisms. The bane of global mode warp models, that forces are simply too weak to overcome differential precession near the edge, has become a strength in our mechanism: the weak coupling of the outer edge creates the warp. Furthermore, the gradual settling of the warm disk avoids any winding problem.

The massive disk can warp slightly, but is largely rigid because of both gravitational restoring forces and radial pressure. The size and lifetime of the warp in the massive disk are probably somewhat overestimated because our numerical method does not yield the full gravitational restoring force. This worry does not affect the conclusions about the warp in the extended $\mathrm{H}$ I layer, which has very little mass and rigidity.

We have deliberately adopted an almost spherical halo in order to show that warps can be formed without misaligned density distributions. Rotating halos are likely, of course, to be slightly flattened also, in which case the disk will respond to both types of forcing. This will lead to warps that precess, $\mathrm{H}$ I layers that do experience forces, and so on. Studies of these cases will be reported in a future paper.

As noted above, we expect the net angular momentum of the halo to be revised as material continues to straggle in long after the main galaxy has reached maturity. Every change to the halo's spin vector can be expected to affect the disk through friction, even if the arriving material is torn apart at large distance by the tidal field of the host galaxy. Our picture is similar to that proposed by Ostriker \& Binney (1989), but they envisage warps as being driven from the outside by a misalignment of the inner disk with the flattened outer halo. In practice, both mechanisms must be inextricably linked. Ongoing infall makes it hardly surprising that warps are detected in most disks.

Conversations with Scott Tremaine and Alar Toomre and the report of the referee, James Binney, were most helpful. The authors wish to thank the Isaac Newton Institute, Cambridge, England for their hospitality for part of this project. This work was supported by NSF grant AST 96/17088 and NASA LongTerm Space Astrophysics grant NAG5-6037.

\section{REFERENCES}

Barnes, J., \& Efstathiou, G. 1987, ApJ, 319, 575

Binney, J. 1992, ARA\&A, 30, 51

Binney, J., Jiang, I.-G., \& Dutta, S. 1998, MNRAS, 297, 1237

Binney, J., \& Tremaine, S. 1987, Galactic Dynamics (Princeton: Princeton Univ. Press)

Bosma, A. 1991, in Warped Disks and Inclined Rings around Galaxies, ed. S. Casertano, P. D. Sackett, \& F. H. Briggs (Cambridge: Cambridge Univ. Press), 181

Briggs, F. H. 1990, ApJ, 352, 15

Debattista, V. P., \& Sellwood, J. A. 1998, ApJ, 493, L5

Dekel, A., \& Shlosman, I. 1983, in IAU Symp. 100, Internal Kinematics and Dynamics of Galaxies, ed. E. Athanassoula (Dordrecht: Kluwer), 187

Dubinski, J., \& Kuijken, K. 1995, ApJ, 442, 492

Hunter, C., \& Toomre, A. 1969, ApJ, 155, 747

Jiang, I.-G., \& Binney, J. 1999, MNRAS, submitted (astro-ph/9807161)

Kalnajs, A. J. 1971, ApJ, 166, 275
Kuijken, K. 1991, ApJ, 376, 467

Lynden-Bell, D., \& Ostriker, J. P. 1967, MNRAS, 136, 293

May, A., \& James, R. A. 1984, MNRAS, 206, 691

McGlynn, T. A. 1984, ApJ, 281, 13

Nelson, R. W., \& Tremaine, S. 1995, MNRAS, 275, 897

Ostriker, E. C., \& Binney, J. 1989, MNRAS, 237, 785

Quinn, T., \& Binney, J. 1992, MNRAS, 255, 729

Raha, N., Sellwood, J. A., James, R. A., \& Kahn, F. D. 1991, Nature, 352, 411

Sparke, L. S., \& Casertano, S. 1988, MNRAS, 234, 873

Steinmetz, M., \& Bartelmann, M. 1995, MNRAS, 272, 570

Toomre, A. 1983, in IAU Symp. 100, Internal Kinematics and Dynamics of Galaxies, ed. E. Athanassoula (Dordrecht: Kluwer), 177

van Albada, T. S. 1982, MNRAS, 201, 939

Villumsen, J. V. 1982, MNRAS, 199, 493

White, S. D. M. 1983, ApJ, 274, 53 